(C) 1995 Elsevier Science B.V. All rights reserved.

\title{
Sorption of light alkanes on H-ZSM5 and H-Mordenite
}

\author{
F. Eder, M. Stockenhuber and J. A. Lercher
}

University of Twente, Department of Chemical Technology, Christian Doppler Laboratory for Heterogenous Catalysis, 7500 AE Enschede, The Netherlands

\section{ABSTRACT}

The sorption of light $\mathrm{n}$ - and iso-alkanes on H-ZSM5 and H-MOR was studied by means of calorimetry, gravimetry and in situ infrared spectroscopy. Alkanes sorb preferentially localized on the strong Brønsted acid sites of H-ZSM5 and H-MOR. Two n-alkane, but only one iso-alkane molecule can be simultaneously accommodated on the Brønsted acid sites of H-ZSM5. In contrast, only one alkane molecule interacts with a strong Brønsted acid site of H-MOR, and, moreover, only a third of these sites are strongly interacting at all with alkanes because of steric reasons. For both zeolites, the heat of adsorption increases with increasing size of the hydrocarbon. The linear relationship between the enthalpies and the entropies of sorption suggests that the molecular motions of the sorbed molecules are restricted in proportion to the strength of interaction with the zeolite.

\section{INTRODUCTION}

As zeolite catalysts are widely used for conversion of hydrocarbons in petrochemical industry, transport, sorption and reaction of such molecules in acidic molecular sieves has attracted significant interest [1]. Several concepts have been proposed to describe the interaction of hydrocarbons with zeolites and, in particular, with the acid sites. Such models either emphasize the local properties of the zeolites to explain the sorption behavior $[2,3,4]$ or use primarily global properties $[5,6]$ for the same purpose.

Hydrocarbon sorption is usually thought to depend more on global than on local properties of the zeolite. With respect to this point, Derouane et al. $[5,6]$ described sorption and diffusion in dependence of the relation between the size of the sorbed molecules and the size and shape of the zeolite pores. It was concluded that the strength of interaction is high when the size of the sorbed molecule and the pore size of the zeolite are similar. For a given zeolite structure the chemical composition, i.e., the polarizability, which is a consequence of it, also influences markedly the strength of interaction between the hydrocarbon and the molecular sieve [7,2]. Mortier et al. $[3,4]$ identified the average polarization of the lattice, i.e., the averaged softness or hardness as the further important factor, that influences the hydrocarbon sorption. The softer, the less polarized the lattice of a molecular sieve is, the stronger is the interaction with alkane molecules. Localized sorption between the apolar alkane and the zeolite occurs as the hydroxyl group induces a dipole in the hydrocarbon. Thus, the strength of the interaction will be determined by the acid strength of the hydroxyl group and the polarizability of the $\mathrm{CH}$ groups of the alkanes. The current communication addresses the description of the interaction of alkanes on acidic ZSM5 and MOR. Gravimetry, calorimetry and i.r. spectroscopy are used as the main experimental means. 


\section{EXPERIMENTAL}

\subsection{Material}

$\mathrm{H}-\mathrm{MOR}(\mathrm{Si} / \mathrm{Al}=10)$ is a dealuminated mordenite obtained from the Japanese Catalysis Society (for a detailed charcterization see ref [8]). Dealumination was carried out by a procedure described previously [9]. H-ZSM5 ( $\mathrm{Si} / \mathrm{Al}=35$ ) was supplied by MOBIL Corp.

\subsection{Gravimetry and calorimetry}

The measurements were performed with a SETARAM TG-DSC 111 instrument. A quantity of about $15 \mathrm{mg}$ of the $\mathrm{NH}_{4}$-form of the samples was charged into the quartz sample holder of the balance and transferred into the hydrogen form by heating in vacuum to $823 \mathrm{~K}$ for one hour. After cooling to $323 \mathrm{~K}$, the alkanes were stepwise introduced into the closed system and equilibrated with the surface. The equilibration was confirmed by observing the heat flow and the changes in weight. If significant changes were not observed the system was considered to be equilibrated. The experiments were carried out in the pressure range from $10^{-3}$ to $13 \mathrm{mbar}$. The pressures were recorded with a BARATRON pressure transducer. The adsorption isotherms of all the hydrocarbons were determined simultaneously with the differential enthalpies of adsorption. The isotherms were fitted to a sum of Langmuir type adsorption isotherms taking into account the differences in adsorption stoichiometries. The concentration of acid sites used in the calculations was taken from ammonia adsorption from ref.[10]. From the equilibrium constants and the heats of adsorption, entropies of adsorption were calculated.

\subsection{Infrared spectroscopy}

The studies were performed with a BRUKER IFS-88 spectrometer. The zeolites were pressed to self supporting wafers and introduced into the heatable sample holder of a vacuum cell. The samples were activated by heating in vacuum $\left(\mathrm{p}<10^{-6} \mathrm{mbar}\right)$ to $823 \mathrm{~K}$ and cooled to adsorption temperature. The sorption experiments were carried out from $303 \mathrm{~K}$ to $373 \mathrm{~K}$ and in pressure range from $10^{-3} \mathrm{mbar}$ to $3 \mathrm{mbar}$. The alkane partial pressure was raised stepwise and after every pressure increase equilibration was observed by time resolved infrared spectroscopy. The decrease in intensity of the $\mathrm{OH}$ stretching vibrations band of the Bronsted acid sites $\left(3610 \mathrm{~cm}^{-1}\right)$ was used to determine the coverage of the acid sites. The integral intensities of the CH-stretching (2800-3000 $\left.\mathrm{cm}^{-1}\right)$ vibrations of the adsorbed alkanes (calibrated by gravimetric measurements) were used to determine the amounts adsorbed.

\section{RESULTS}

\subsection{Adsorption on H-ZSM5}

The values for the sorption enthalpy of the hydrocarbons are compiled in Table 1. For H-ZSM5, the sorption enthalpies of n-alkanes were constant up to a coverage of two molecules per Brønsted acid site. At higher coverages, the values decreased (see Fig. 1). For iso-alkanes, however, the heat of adsorption decreased already at a coverage of one iso-alkane molecule per acid site.

Infrared spectra show that only the band of the Brønsted acid Si-OH-Al groups at $3610 \mathrm{~cm}^{-1}$ decreased in intensity upon adsorption of the hydrocarbons. At sufficiently high partial pressures (e.g., 1 mbar of $n$-hexane at 303K) the hydroxyl band disappeared indicating that all Brønsted acid sites of H-ZSM5 interacted with the alkanes.

At low coverages the uptake of hydrocarbons corresponded exactly to the fraction of free 


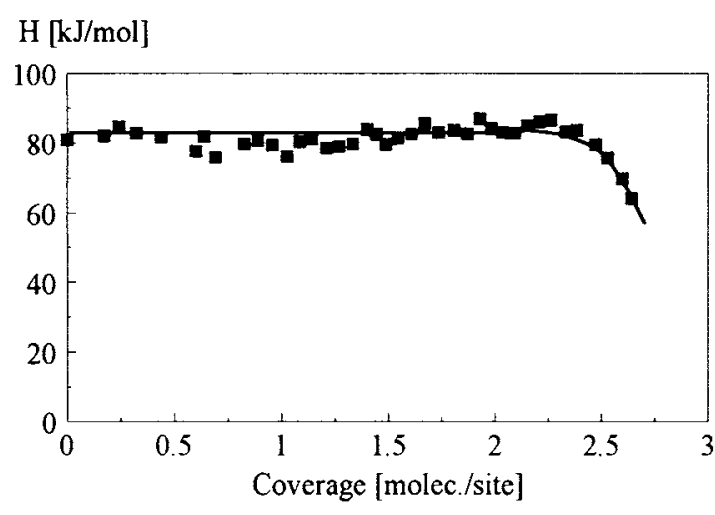

Figure 1 Adsorption of $\mathrm{n}$-hexane on H-ZSM5 hydroxyl groups that disappeared. This suggests that one molecule is adsorbed per acid site. When more than $30 \%$ of the acid sites were covered, the number of $n$ alkanes adsorbed per acid site exceeded markedly this stoichiometry indicating that hydrocarbon molecules adsorbed on Si-OH-Al groups already interacting with one alkane molecule, or were adsorbed delocalized, while other Brønsted acid sites were still free.

Upon sorption of the alkane molecules, a broad band characteristic of hydrogen bonded (perturbed) $\mathrm{OH}$ groups appeared

at lower wavenumbers. The wavenumber difference between this band and the band for the free $\mathrm{Si}-\mathrm{OH}-\mathrm{Al}$ groups increased with increasing size of the hydrocarbons indicating an increase in the strength of the direct interaction with the acid sites [11]. For a given n-alkane the wavenumber of the band of the perturbed $\mathrm{OH}$, however, also decreased with increasing coverage. Because of the uniform acid strength of the hydroxyl groups in H-ZSM5 [10] and in agreement with observed stoichiometries of adsorption, we attribute this red shift of the perturbed $\mathrm{Si}-\mathrm{OH}-\mathrm{Al}$ group to an increasing fraction of $\mathrm{OH}$ groups interacting with more than one molecule. Such multiple interactions enhance the perturbation. The asymmetric form of the band of the perturbed hydroxyl groups allowed to separate two contributions, i.e., the band between 3570 and $3500 \mathrm{~cm}^{-1}$ (depending on the substance adsorbed) attributed to sorption of one and the band between $3445 \mathrm{~cm}^{-1}$ and $3485 \mathrm{~cm}^{-1}$ attributed to sorption of two molecules per acid site. The 1:1 stoichiometry contributed most at low coverages, the 2:1 stoichiometry at high coverages. It should be emphasized that such a sorption behavior was only observed for $\mathrm{n}$-alkanes on $\mathrm{H}-\mathrm{ZSM}$ 5. The band of the perturbed $\mathrm{OH}$ groups after adsorption of iso-alkanes had a symmetric form and the absorption maximum was independent of the coverage.

Table 1

Heats of adsorption on and maximum sorption capacity at 323K on H-ZSM5 and H-MOR

\begin{tabular}{lcccc}
\hline & \multicolumn{3}{c}{$\mathrm{H}-\mathrm{MOR}$} \\
\cline { 2 - 5 } & $\Delta \mathrm{H}_{\mathrm{ads}}\left[\mathrm{kJmol}^{-1}\right]$ & $\begin{array}{c}\text { Loading } \\
{[\mathrm{mmol} / \mathrm{g}]}\end{array}$ & $\Delta \mathrm{H}_{\text {ads }}\left[\mathrm{kJmol}^{-1}\right]$ & $\begin{array}{c}\text { Loading } \\
{[\mathrm{mmol} / \mathrm{g}]}\end{array}$ \\
\hline propane & -46 & 1.47 & -41 & 0.9 \\
n-butane & -58 & 1.39 & -50 & 0.75 \\
iso-butane & -52 & 0.67 & -52 & 0.64 \\
n-pentane & -70 & 1.28 & -59 & 0.65 \\
iso-pentane & -64 & 0.71 & -61 & 0.61 \\
n-hexane & -82 & 1.24 & -69 & 0.61 \\
\hline
\end{tabular}




\subsection{Sorption on H-MOR}

The values of the enthalpies of sorption of the alkanes on H-MOR are compiled in Table 1. Up to a coverage of 0.35 to 0.45 alkane molecules per strong acid site the heat of adsorption on H-MOR was nearly constant. At higher coverages the heat of adsorption decreased suggesting that only about $40 \%$ of the acid sites are accessible to alkane molecules. The intensity of the band of the Bronsted acidic Si-OH-Al group at $3610 \mathrm{~cm}^{-1}$ decreased upon the sorption of alkanes to a minimum of two third of its original value. The asymmetric form of this band suggests the existence of two kinds of acid sites characterized by two bands with maxima at $3610 \mathrm{~cm}^{-1}$ and $3590 \mathrm{~cm}^{-1}$, respectively (see Fig.2). They do not differ in acid strength (as seen by sorption of ammonia [10])

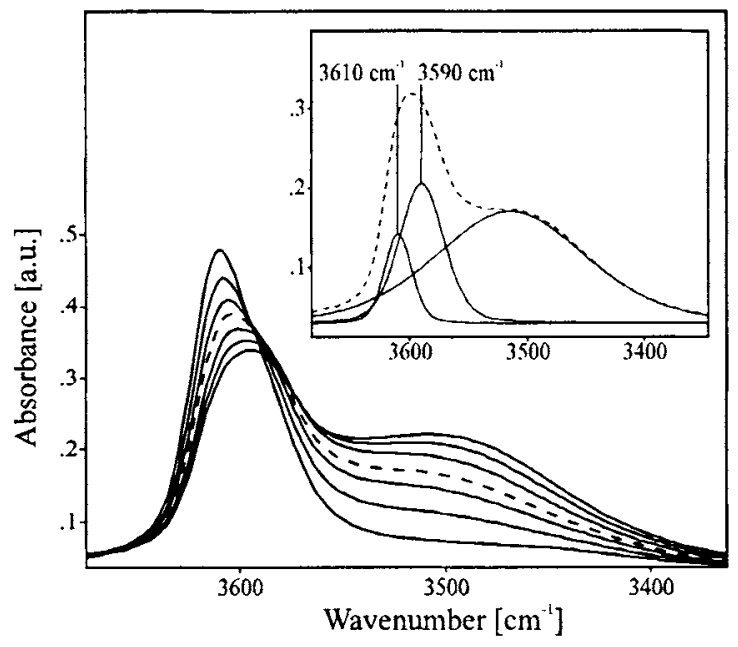

Figure 2. Adsorption of n-hexane on H-MOR. The lines correspond to increasing equilibrium pressures of $n$ hexane. but in location and environment. The fraction of the acid sites characterized by the band at $3610 \mathrm{~cm}^{-1}$ that disappeared upon hydrocarbon sorption caused a band characteristic of hydrogen bonding some 90 to 115 $\mathrm{cm}^{-1}$ lower than the wavenumber of the free $\mathrm{Si}-\mathrm{OH}-\mathrm{Al}$ group. As with $\mathrm{H}$ ZSM5, the wavenumber decreased with the chain length of the adsorbed molecules. In contrast to the observations on H-ZSM5 the shift was independent of the coverage for a given alkane. The concentration of hydrocarbon molecules adsorbed corresponded well to the fraction of hydroxyl groups in interaction. Thus, we conclude that one alkane is adsorbed per accessible Brønsted acid site.

\section{DISCUSSION}

I.r. spectroscopy indicates that most of the hydrocarbons adsorbed are bound via hydrogen bonds to the Brønsted acid sites of the two zeolites studied. This bonding involves induction of polarity in the alkane by the hydroxyl group. The strength of the bonding (indicated by the difference of the band of the perturbed and the unperturbed $\mathrm{OH}$ groups [11]) depends upon the extent of the polarization of hydrocarbon $\mathrm{C}-\mathrm{H}$ bonds and the strength of the Bronsted acidic $\mathrm{OH}$ group. The difference in wavenumbers between the free and the perturbed $\mathrm{Si}-\mathrm{OH}-\mathrm{Al}$ group was at least for the larger hydrocarbons quite similar for H-ZSM5 and H-MOR. Thus, we conclude that the Si-OH$\mathrm{Al}$ groups in the H-ZSM5 and H-MOR investigated have a similar acid strength with respect to hydrocarbons. Previous characterization by sorption of strong bases [10] indicated also a rather similar acid strength of the two zeolites.

With increasing size of the sorbed alkanes the strength of hydrogen bonding to Brønsted acid sites (monitored via the difference in wavenumbers of perturbed and unperturbed Bronsted acid 
$\mathrm{OH}$ groups) and the sorption enthalpy increased. This indicates that the polarizability of the alkanes increases with increasing carbon numbers of the alkanes. It is striking, however, that in contrast to the strength of the direct hydrogen bonding to the Bronsted acidic OH groups, the increase in enthalpy is more pronounced with H-ZSM5 than with H-MOR. In absolute terms, the heats of adsorption of all $\mathrm{n}$-alkanes investigated was at least 5 to $13 \mathrm{~kJ} / \mathrm{mol}$ higher on $\mathrm{H}$-ZSM5 than on H-MOR. This indicates that the main reason for the higher sorption enthalpy with H-ZSM5 are the more intense lateral interactions of the hydrocarbons with the lattice. Such an interpretation agrees well with previous communications reporting that the van der Waals interaction between alkane molecules and the lattice of zeolites increases with the chain length of the sorbed alkanes $[5,12,13]$. We would like to attribute this higher strength of lateral interactions between the alkanes and the zeolite lattice to the smaller pore size of H-ZSM5 $[5,6]$ and/or to the higher polarizability of its lattice ("softer character" of H-ZSM5 $[3,4]$ ) which should cause stronger interactions with soft bases like alkanes.

A linear relationship between the enthalpies and the entropies of sorption was found suggesting one common adsorption structure for all $n$-alkanes and that the molecular motions of the sorbed molecules are restricted in proportion to the strength of interaction with the zeolite. Different slopes for H-MOR and H-ZSM5 suggest that the structure of the zeolite has a distinct influence on the molecular motions of the adsorbed molecule. The same enthalpy of adsorption results in higher entropy of adsorption for H-ZSM5 compared with that for H-MOR indicating again a more pronounced interaction of the alkanes with the lattice H-ZSM5.

With respect to the sorption stoichiometry at higher loadings, a unique behavior was found for the n-alkanes in H-ZSM5. n-Alkanes are adsorbed localized in a stoichiometry of up to two molecules per acid site of H-ZSM5. Indeed, the maximum sorption capacity of iso-alkanes on $\mathrm{H}$ ZSM5 is approximately a factor of two smaller than the maximum sorption capacity of n-alkane molecules. In contrast, the gravimetric and calorimetric results and the correlation between the intensity of the $\mathrm{CH}$ i.r. bands of the sorbed alkanes and $\mathrm{OH}$ band of the acid sites indicate that the $\mathrm{n}$ - and iso-alkanes are sorbed in a stoichiometry of one molecule per accessible acid site in H-MOR. Therefore, the maximum uptake of n-alkanes in H-MOR was significantly lower than in H-ZSM5. Because localized sorption of two alkane molecules per acid site was not observed for any of the large pore zeolites, we conclude that this unique sorption of n-alkanes in H-ZSM5 is due to the excellent fit between two adlineated n-alkanes and the channels or the intersections of the zeolite. This view is supported by the fact that the adsorption enthalpy was the same, if one or two molecules were adsorbed. As the addition of a further molecule to an already sorbed molecule must lead necessarily to a lower strength of the direct interaction, the constant enthalpies point to a strong stabilization of the sorption complex by the surrounding. This seems possible for the medium size pore ZSM5, but not for the large pore H-MOR.

Finally, as shown by i.r. spectroscopy, all Brønsted acid sites of H-ZSM5, but only one third of the acid sites of H-MOR strongly interacted with the alkane molecules. Because the acid sites showed uniform acid strength, we conclude that the alkanes cannot interact with some the acid sites in H-MOR because of sterical reasons. These acid sites are concluded to be located in the side pockets of the H-MOR channels $[14,15]$. The entrance of the side pocket has an diameter of $0.39 \times 0.57 \mathrm{~nm}$, the opening at the end of the pocket has a diameter of $0.39 \times 0.28 \mathrm{~nm}$, which is substantially smaller than the aperture of the large pores $(0.67 \times 0.7 \mathrm{~nm})$. The critical diameter of linear alkanes was calculated to $0.45 \mathrm{~nm}[16,17]$. Hence acid sites in the side pockets must be very difficult to reach for the alkane molecules. A similar effect was observed for the adsorption of benzene and cyclohexane on H-MOR [18]. 


\section{CONCLUSIONS}

The present report shows the distinct differences between sorption of light alkanes on zeolites H-ZSM5 and H-MOR. While all Brønsted acid sites of H-ZSM5 are accessible for alkane molecules, only one third of the acid sites of H-MOR (i.e., the fraction located in the main channels) strongly sorb these molecules. With H-ZSM5 the n-alkanes are sorbed locally at the acid sites in a maximal stoichiometry of two molecules per acid sites, the bulkier iso-alkanes in a one to one stoichiometry. With H-MOR one molecule of $\mathrm{n}$ - and iso-alkanes is sorbed locally per accessible acid site. This localized direct interaction (hydrogen bonding) between the alkanes and the strong Brønsted acid sites is of similar strength with both zeolites. The hydrogen bonding occurs as the charge at the Brønsted acid site induces a dipole in the alkane. The interaction gains strength with increasing chain length of the hydrocarbon due to (i) enhanced polarization and (ii) stronger lateral interactions of the hydrocarbons with the lattice oxygen. For a given hydrocarbon H-ZSM5 forms tighter bound surface complexes than H-MOR reflected in a larger heat release and a more pronounced entropy loss due to sorption.

\section{ACKNOWLEDGEMENTS}

Financial support of the Christian Doppler Society is gratefully acknowledged.

\section{REFERENCES}

1. W.O. Haag, Stud. Surf. Sci. Cat., 84 (1994) 1375.

2. A. Corma, G. Sastre, R. Viruela and C. Zicovich-Wilson, J. Catal., 136 (1992) 521.

3. K.A. Genechten and W. Mortier, Zeolites, 8 (1988) 273.

4. W. Mortier, Stud. Surf. Sci. Catal., 37 (1988) 253.

5. E.G. Derouame, J.B. Janos, C.Fernandez, Z. Gabelica, E. Laurent and P. Maljean, Appl. Catal., 40 (1988) L1.

6. E.G. Derouane, J.-M. Andre and A.A. Lucas, J. Catal., 110 (1988) 58.

7. R.G. Pearson, J. Am. Chem. Soc., 85 (1963) 3533.

8. M. Sawa, M. Niwa and Y. Murakami, Zeolites, 10 (1990), 532.

9. G.I. Kapustin, T.R. Brueva, A.L. Klyachko, S. Beran and B. Wichterlova, Appl. Catal. $42,(1988) 239$

10. M. Stockenhuber and J.A.Lercher, in preparation.

11. M.L. Hair and W. Hertl, J. Phys. Chem., 74 (1970) 91.

12. J.O. Titiloye, S.C. Parker, F.S. Stone and C.R.A. Catlow, J. Phys. Chem. 95 (1991) 4038.

13. B. Smit and J.I. Siepmann, J. Phys. Chem., 98 (1994) 8442.

14. W.M. Meier, Z. Kristallogr. 115 (1961) 439.

15. K. Shiokawa, M. Ito and K. Itabashi, Zeolites, 9 (1989) 170

16. E.G. Derouane, "Intercalation Chemistry", Academic Press, New York, (1984) 101.

17. R.M. Barrer, "Zeolites: Science and Technology", NATO ASI Series E, Martin Nijhoff, The Hague, 80 (1984) 227.

18. V.L. Zholobenko, M. A. Makarova and J. Dwyer, J. Phys. Chem. 97 (1993) 5962. 